\title{
Plasma Diagnostics
}

\section{P.D. Morgan, R. Behn and M.R. Siegrist, Lausanne}

(EPFL Research Centre for Plasma Physics)

During the past 18 years, lasers have played an increasingly important role in the diagnostics of laboratory plasmas, in particular those studied for thermonuclear applications. Today, the laser is widely regarded as being the most versatile single tool for making measurements on these plasmas. Among the various parameters that have been determined using lasers are: electron density $n_{\mathrm{e}}$, electron temperature $T_{e^{\prime}}$ ion temperature $T_{i}$, impurity concentration, microturbulence level and magnetic field strength $\mathbf{B}$. In this article, the basic principles of the most powerful laser diagnostic technique, viz. Thomson scattering, are reviewed.

\section{Scattering - the Principles}

Consider a plane-polarised e.m. wave incident on a free electron at rest. The electron will oscillate at the wave angular frequency $\omega_{0}$, parallel to the direction of the electric vector. The electron then reradiates at the frequency $\omega_{0}$. At moderate field strengths, the total cross section for this scattering process is the Thomson scattering cross section:

$\sigma_{\mathrm{T}}=(8 \pi / 3) r_{\mathrm{e}}^{2}=6.65 \times 10^{-25} \mathrm{~cm}^{2}$ where $r_{\mathrm{e}}$ is the classical electron radius.

If the electron is moving with a velocity $v_{e}$ it encounters the wave at a Dopplershifted frequency:

$\omega_{\mathrm{D}}=\omega_{0}-\mathbf{k}_{\mathrm{o}} \cdot \mathbf{v}_{\mathrm{e}^{\prime}}$ where $\mathbf{k}_{\mathrm{o}}$ is the propagation vector of the incident wave. The scattered radiation emitted by the electron will have the frequency $\omega_{D}$ in its moving coordinate frame. If this radiation, with wavenumber $\mathbf{k}_{\mathrm{s}^{\prime}}$ is observed at an angle $\theta$ with respect to $\mathbf{k}_{0}$, Fig. 1, a further Doppler shift will occur, corresponding to the velocity component of the electron along the direction of $\mathbf{k}_{\mathrm{s}}$. The scattered radiation will have the frequency:

$$
\begin{aligned}
\omega_{\mathrm{s}} & =\omega_{\mathrm{D}}+\mathbf{k}_{\mathrm{s}} \cdot \mathbf{v}_{\mathrm{e}} \\
& =\omega_{0}-\mathbf{k} \cdot \mathbf{v}_{\mathrm{e}}
\end{aligned}
$$

where $\mathbf{k}=\mathbf{k}_{\mathrm{o}}-\mathbf{k}_{\mathrm{s}}$

The observed frequency shift:

$$
\omega=\omega_{\mathrm{o}}-\omega_{\mathrm{s}}
$$

is due to the component of electron velocity along $\mathbf{k}$, and has the magnitude $\mathbf{k} \cdot \mathbf{v}_{\mathrm{e}}$. Since, for non-relativistic cases, $\left|\mathbf{k}_{\mathrm{s}}\right| \cong\left|\mathbf{k}_{\mathrm{o}}\right|$, $|\mathbf{k}|=2\left|\mathbf{k}_{\mathrm{o}}\right| \sin (\theta / 2)=\left(4 \pi / \lambda_{\mathrm{o}}\right) \sin (\theta / 2)$ where $\lambda_{0}$ is the wavelength of the incident radiation.

In a plasma, because of the large mass difference between electrons and ions it is the former that are the dominant scatterers, since they are influenced far more by the e.m. wave. For uniform charge distribution, the total power scattered would be almost zero due to interference between contributions of opposite phase. However, electron density deviations, $\delta n_{\mathrm{e}}$, occur in all plasmas due to random thermal fluctuations and coherent collective motions. These bunched electrons emit constructively an intensity proportional to $\left(\delta n_{\mathrm{e}}\right)^{2}$. If $\delta n_{\mathrm{e}}$ is random, its magnitude varies as $\sqrt{ } n_{\mathrm{e}}$. Hence the scattered intensity from a thermal plasma is proportional to its density $n_{e}$. Scattering from a wave of amplitude $\delta n_{\mathrm{e}} / n_{\mathrm{e}}$ would have an intensity proportional to $\left(\delta n_{e}\right)^{2}$.

Scattering from a plasma can be regarded as a three-wave interaction. An e.m. wave of frequency $\omega_{0}$ and propagation vector $\mathbf{k}_{0}$, interacts with a density fluctuation, characterised by $\omega$ and $\mathbf{k}$, to produce a scattered wave of frequency $\omega_{\mathrm{s}}$ and vector $\mathbf{k}_{\mathrm{s}}$. Momentum and energy conservation have to be satisfied, eqns. (4) and (5). In any plasma there exists a large range of scale lengths on which the electron density is fluctuating. By a judicious choice of $\lambda$ 。 and $\theta$, eqn. (6), the experimenter can determine the wavelength, $2 \pi /|\mathbf{k}|$, of the fluctuations that will be revealed in a scattering measurement. This is the fundamental concept of laser scattering.

It is the ratio of the fluctuation wavelength to the plasma electron Debye length, $\lambda_{\mathrm{D}}$, that determines the spectrum of the scattered radiation. The Debye length defines the range over which the Coulomb field of a charge imbalance extends; the flow of electrons which counteracts this inequality shields the field and limits its range. In c.g.s. units $\lambda_{\mathrm{D}}=6.90 \sqrt{ }\left(T_{\mathrm{e}} / n_{\mathrm{e}}\right)$. If $\alpha=\left(|\mathbf{k}| \lambda_{\mathrm{D}}\right)^{-1}<<1$, the fluctuation length is much shorter than the Debye length and the observed spectrum reflects scattering off random density fluctuations - incoherent scattering. For a plasma with a Maxwellian electron velocity distribution, the spectrum, Fig. 2a, is a Gaussian centred on the laser frequency, as electrons are moving in all directions with respect to k. The half width of the spectrum is proportional to $\sqrt{ } T_{e}$. For the case of $\theta=90^{\circ}$, $\lambda_{0}=6943 \AA$ and $T_{e}=5 \times 10^{6} \mathrm{~K}$ the width of the spectrum would be $730 \AA$ FWHM. The line width of the incident light would be $\leq 0.5 \AA$.

In the case of $\alpha>>1$, the length $2 \pi / \mid \mathbf{k}$ is much longer than $\lambda_{\mathrm{D}}$ and scattering off collective fluctuations is observed, Fig. $2 b$, e.g. due to scattering off electron clouds which are Debye shielding the ions, and electron bunches propagating in plasma waves. The half width of the central feature is proportional to $\sqrt{ } T_{i}$, if the plasma is in thermal equilibrium. The two shoulders arise from scattering off ion-acoustic waves, while the two detached satellites are due to scattering off electron plasma waves. Because of the dependence of the thermal velocity on particle mass, at a given temperature the frequency scale for the ion feature is at least 43 times narrower than that for the electron feature - this number being the square root of the mass ratio of the proton to the electron.

As the Thomson cross section is so small, scattering is an extremely weak interaction. This is excellent in that the plasma is not pertubed by making a measurement, but an extremely powerful source of radiation is needed to observe scattering. The scattered power, $P_{\mathrm{s}^{\prime}}$ may be related to the incident power, $P_{0^{\prime}}$, by:

$$
P_{\mathrm{s}}=P_{0} n_{\mathrm{e}} / \mathrm{d} \Omega r_{\mathrm{e}}^{2} S(\mathbf{k}, \omega)
$$

where $/$ is the plasma length from which scattered radiation is collected, $d \Omega$ is the collection solid angle and $S(\mathbf{k}, \omega)$ is the spectral density function. The last quantity defines the scattered power versus frequency distribution for a given $\mathbf{k}$.

If we take values typical of a tokamak experiment to observe incoherent scattering: $n=3 \times 10^{13} \mathrm{~cm}^{-3}, l=1 \mathrm{~cm}, \mathrm{~d} \Omega=5 \times$ $10^{-3} \mathrm{str}$ and, integrating over all frequencies, $S(\mathbf{k}, \omega)=1$, the ratio $P_{\mathrm{s}} / P_{0} \cong 10^{-14}$, which is minute. Consequently, incoherent scattering from a laboratory plasma was not observed before the advent of the laser. However, even with such a powerful source, extreme care has to be taken or radiation not scattered by the plasma will fall onto the detector and obscure the signal. This stray radiation is mainly due to scattering from optical components in the laser beam and diffuse reflection from the walls of the plasma chamber.

\section{Scattering Applications}

The first reports on the detection of incoherent scattering from a plasma were

Fig. 2 - Electron and ion features for scattering from (a) incoherent and (b) collective density fluctuations.
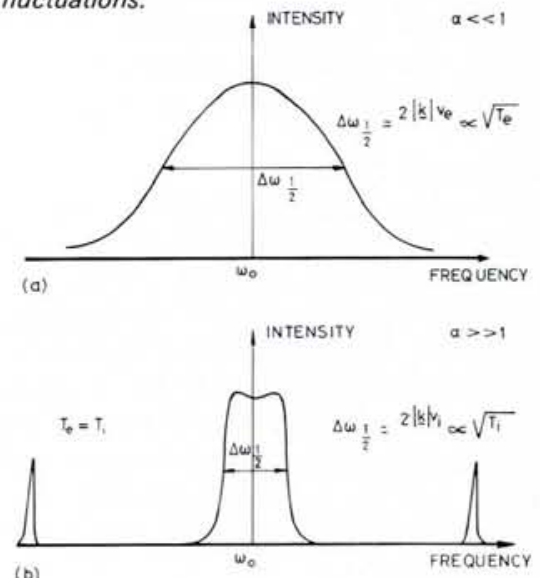


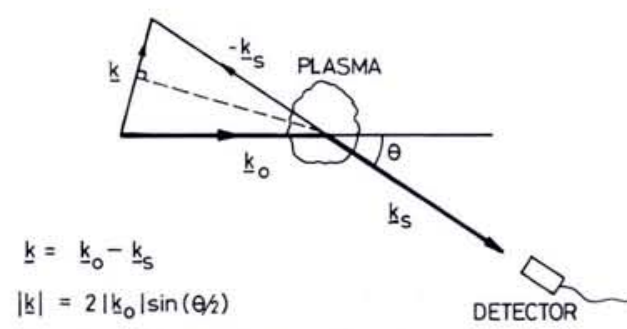

Fig. 1 - Vector diagram showing relationship between $\mathrm{k}_{\mathrm{o}}, \mathrm{k}_{\mathrm{s}}$ and $\mathrm{k}$.

Fig. 3 - Schematic diagram of apparatus to measure $T_{e}$ on the Lausanne TCA tokamak. Using two pulses, $T_{e}$ is measured at two separate times during one tokamak discharge. Inset - typical intensity / wavelength distribution for the 10 channels.

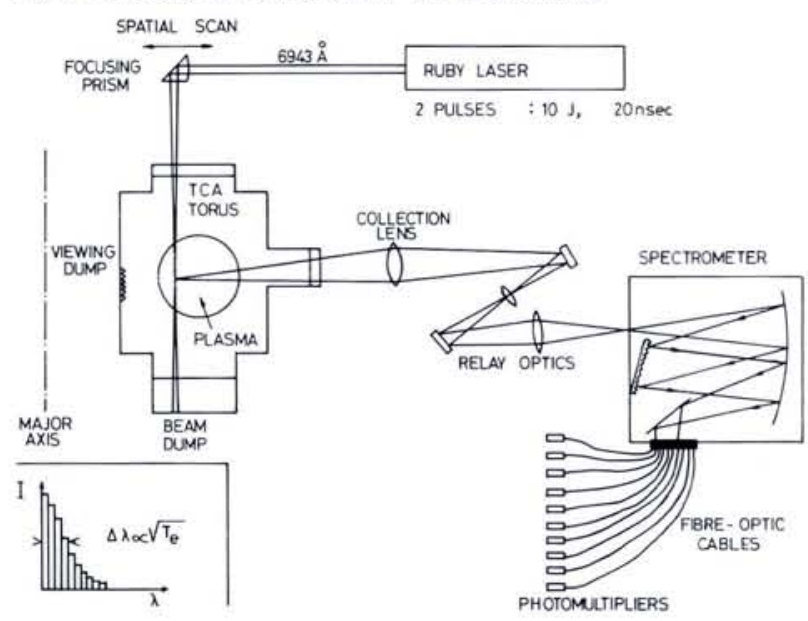

\section{W IN THE $9 R 8$ LINE TO PUMP YOUR F.I.R. WAVEGUIDE...}

is provided by our model 6000 , highly stable tunable $\mathrm{CO}_{2}$ laser. Up to $50 \mathrm{~W}$ output power (TEMoo, single frequency) will be achieved in the strongest lines between $9 \mu \mathrm{m}$ and $11 \mu \mathrm{m}$.

Operational modes: C.W., chopped and pulsed, optional Q-switch. A F.I.R. waveguide is available as accessory.

For more information:

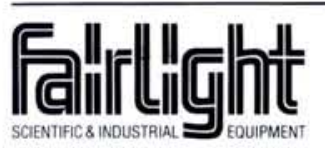

Fairlight Scientific \& Industrial Equipment P.O. Box 40553006 AB Rotterdam the Netherlands tel.: 010-333418 tlx.. 21252 fairl nl made in 1963 by Thompson and Fiocco and by Funfer et al. In 1964 Davies and Ramsden presented unambiguous results on the electron velocity distribution in a theta-pinch plasma. Peacock et al. in 1968 measured $T_{e}$ in the Russian T-3 tokamak, the first scattering measurement on such a plasma. In all those experiments a pulsed ruby laser was used as source. Today, Thomson scattering using such a laser is a routine technique for measuring $T_{e}$ in laboratory plasmas.

The first observation of collective scattering from a thermal plasma was made in 1964 by DeSilva et al. They recorded the spectrum of ruby laser light scattered at $10^{\circ}$ from a theta-pinch plasma. Further measurements, using similar apparatus, were performed in 1964 by Kunze et al. and in 1966 by Ramsden et al. In 1969 Baconnet et al. measured $T$ in a plasma focus device, a high-density z pinch, and in 1974, Forrest and Peacock repeated the exercise. The latter were able to show that neon ions present as an impurity in the plasma had a temperature that was an order of magnitude higher than that of the majority of deuterium ions. Evans showed theoretically in 1970 that even concentrations as low as a few p.p.m. of highly stripped ions can be detected by collective scattering. The high charges on these ions attract large clouds of shielding electrons, causing significant departures from uniformity. Also, due to their greater mass, the velocities of the impurity ions are usually much lower than those of the majority ions. Hence, the impurity appears as an intense spike superposed on the main ion feature.

In a tokamak plasma, because of the relatively high temperature and low density, the Debye length is large, typically of the order of $30 \mu \mathrm{m}$. To satisfy the condition for observing collective scattering, $\alpha>1$, using an optical laser the angle of observation $\theta$ has to be very small, usually $\leq 0.1^{\circ}$. Since scattered light is collected from along the whole laser beam path in the plasma, spatial resolution is non-existent. Also, as the collection optics are almost pointing down the main laser beam, stray light is a severe problem. A way of overcoming these difficulties is to use a laser emitting at far-infrared wavelengths, typically several hundred $\mu \mathrm{m}$, so that angles of tens of degrees can be employed. Currently, several groups are engaged in developing far-infrared lasers of performance adequate to measure $T_{\text {in }}$ in tokamak plasmas.

The presence of a magnetic field in a plasma will, under certain conditions, modify the spectrum of scattered radiation. The charged particles will follow the field lines, moving along helical trajectories. If the scattering vector $\mathbf{k}$ is perpendicular to the field $\mathbf{B}$, the electron and ion features are modulated at the electron and ion gyrofrequencies $\left(\omega_{\text {ce }}\right.$ and $\left.\omega_{c i}\right)$, respectively. The envelope of each spectrum is the same as in the absence of the field, but it is broken up into a series of peaks and troughs separated by $\omega_{\mathrm{ce}}$ or $\omega_{\mathrm{ci}}$, as appropriate. If $\mathbf{k}$ and $\mathbf{B}$ are a few degrees out of perpendicular, the modulations are smeared out due to broadening caused by the component of thermal velocity along $\mathbf{k}$. Hence, in principle, the field strength $B$ can be deduced by measuring in an incoherent scattering experiment the separation between peaks, since $\omega_{c e}$ is proportional to $B$. Furthermore, the local field direction can be determined by finding the direction of $\mathbf{k}$ for which modulation is observed. In practice the measurement is very difficult to perform, although it has been successfully applied to a theta-pinch plasma in 1970 by Evans and Carolan and to a tokamak plasma in 1978 by Forrest $e t$ al.

Finally, in the collective scattering regime, microturbulence, which is responsible for anomalous transport and energy loss in toroidal plasma machines, can be studied. Since in a tokamak the fluctuations level $\delta n_{\mathrm{e}} / n_{\mathrm{e}}$ due to microturbulence can reach values as high as 0.01 , which is at least four orders of magnitude higher than the thermal level, high power lasers are not needed - powers of a few tens of $\mathrm{mW}$ to a few tens of $\mathrm{W}$ are adequate. In these plasmas, the spectrum for microturbulence is many orders of magnitude narrower than that for the thermal ion feature. In particular, Slusher and Surko have performed many studies on tokamak plasmas using a c.w. $\mathrm{CO}_{2}$ laser of wavelength 10.6 $\mu \mathrm{m}$. They have measured the level of microturbulence under many conditions, as a function of $\mathbf{k}$ and $\omega$. To obtain good 
spatial resolution, since angles $\theta$ of a few degrees at most can be employed, in 1978 they developed a technique whereby two laser beams intersected in a small plasma volume. Narrow-angle scattering was observed from each beam, but by using a cross-correlation technique only fluctuations common to both were recorded.

BIBLIOGRAPHY

1. Sheffield J., Plasma Scattering of Elec tromagnetic Radiation (Academic Press) 1976.
2. Morgan P.D., Green M.R., Siegrist M.R. and Watterson R.L., "The development of far-infrared lasers for Thomson-scattering measurements on tokamak plasmas", Comments Plas. Phys. Cont. Fusion 5 (1979) 4, p. 141

3. Forrest M.J., Carolan P.G. and Peacock N.J. "Measurement of magnetic fields in a tokamak using laser light scattering", Nature 271 (1978) 5647, p. 718.

4. Surko C.M. and Slusher R.E. " "Study of plasma density fluctuations by the correlation of crossed $\mathrm{CO}_{2}$ laser beams", Phys. Fluids 23 (1980) 12, p. 2425.

\section{Applications to Biology and Medicine}

\section{A. Andreoni, R. Cubeddu and C.A. Sacchi, Milan}

(Quantum Electronics and Electronic Instrumentation Centre of the CNR)

Ever since it first appeared, the laser has been considered a highly promising instrument for biomedical research. As early as 1962, Britton Chance (Johnson Research Foundation) reported, at the annual meeting of the American Biophysical Society, on a study of electron transfer in photosynthetic algae, made with a ruby laser. In the same year, M. Bessis (Ecole Pratique des Hautes Etudes, Paris) published his results on "Irradiation des organites cellulaires à l'aide d'un laser à rubis", and C.J. Campbell (Columbia University, N.Y.) published a paper on the use of "The optical maser as a retinal coagulator".

It will be recalled that the annual conference of the Quantum Electronics Division of EPS, held in Florence in 1979, was devoted to "Lasers in Photomedicine and Photobiology".

Applications of the laser to Biology and Medicine are diverse, exploiting one or more of the special properties of the device and the measuring techniques that have been described in relation to chemistry and other disciplines. But besides being a diagnostic tool, a laser can be used to act on a biological sample. Examples of such applications are the laser activation of photosynthesis, the micro-irradiation of cells and cellular organelles, and, in medicine, the photodynamic action of some drugs, surgery, and photocoagulation.

\section{Biology}

Many structural and conformational properties of the most relevant biomolecules (DNA, RNA, proteins, lipids, etc.) have been studied "in vivo" by means of laser spectroscopy, notably emission spectroscopy performed on suitable dyes that label these biomolecules. In particular, time-resolved fluorescence and polarization have been applied successfully. This is due to the fact that the binding of a dye molecule to a biomolecule induces marked changes in certain fluorescence properties such as the non-radiative decay pathways of its excited state.

Studies on DNA have been made with Acridine dyes which exploit this effect. The DNA-specific staining dyes Proflavine and Quinacrine Mustard (OM) were chosen, because their fluorescence properties depend on the DNA site where they bind. In particular, they intercalate between adjacent base-pairs in the DNA double helix, and have a longer excited-state ( $S_{1}$ ) lifetime when they intercalate two AdenineThymine (AT) base-pairs than when they are bound to sites containing at least one Guanine-Cytosine (GC) base-pair. Although the origins of this property were unknown, the DNA base-pair composition of a number of substances could be determined.

It then proved possible to explain the reasons for the behaviour: these molecules undergo excited-state proton transfer and the rates of proton exchange are influenced by the base-pair composition of the DNA binding site.

Many other experiments have been made in which the same dyes have been used to probe some structural property of the DNA "in vivo", including some on a microscopic scale using a microfluorometer. In this, the exciting laser pulse (generated by a nitrogen-pumped tunable dye laser) is concentrated through a microscope objective down to a spot of $0.5 \mu \mathrm{m}$ diameter; the subsequent fluorescence is detected by a fast photomultiplier ( $0.2 \mathrm{~ns}$ risetime). Among the applications of this instrument, can be mentioned a study of the DNA base-pair composition in single bacteria with $\mathrm{QM}$.

With the same instrument also, experiments in fluorescent-drug microdosimetry, such as the study of cell or tissue uptake of antitumour drugs) can be made. In this, the initial strength of the fluorescence pulse is related to the local amount of drug, while its time evolution is indicative of the physical condition of the drug molecule.
Fluorescent labels find another important application in flow cytometry. In this technique, mammalian cells in suspension are lined up by passing through a special flow chamber, and exposed one at a time to a laser beam. Crossing the beam, each cell produces one or more optical pulses that are related to its DNA (or RNA) content, dimensions and so on. This may allow discrimination between tumour and normal cells, and analysis of the cell cycle or drug effects. It is the only way of making rapid quantitative measurements on large numbers of cells (typically, at a rate of $5 \times 10^{4}$ cells $/ \mathrm{min}$ ), with high statistical precision. Commercial versions of the instrument have been on the market for several years, and are currently used in several hospitals and institutes of biomedical research.

Very recently, we have conducted new experiments, in which Acridines were used as intermediates to act on the DNA, rather than to probe it. The effect is produced via a two-step photoionization of the dye molecule, and results in damage to the DNA base pairs that it intercalates. Different schemes for the interaction of intense laser pulses have been proposed, in order to obtain an overall efficiency of the photoionization process that is dependent on the $S_{1}$ lifetime. The irradiation of $\mathrm{QM}$, bound to synthetic polynucleotides containing only GC or AT bases, with nitrogen laser pulses of length shorter than the $\mathrm{S}$, lifetime of $\mathrm{QM}$ bound to $\mathrm{AT}$, but longer than that of $\mathrm{QM}$ bound to $\mathrm{GC}$, resulted in a destruction of the complex $\mathrm{QM}: \mathrm{GC}$ one fifth that of the complex QM:AT. A much higher contrast (a factor of over 40 ) between the photodamage of the two complexes was obtained using Proflavine (PF) as the staining agent and pulses from two lasers of different wavelengths with a delay between them.

\section{Medical Research}

The laser began to figure in medical research at an early stage, the first application being coagulation in ophthalmology using a ruby laser. This is now done with an argon laser, and is accepted throughout the world as the treatment for retinal detachment and for nonproliferative retinopathy.

After the invention of the $\mathrm{CO}_{2}$ laser, the surgical applications began to increase rapidly. When focused on the surface of any living tissue, the $\mathrm{CO}_{2}$ laser beam is strongly absorbed (in a layer of $\sim 200 \mu \mathrm{m}$ ) by the water contained in the tissue $(80-90 \%)$, and transformed into heat. With an intensity of a few $\mathrm{W} / \mathrm{mm}^{2}$, the tissue fluids boil almost instanteneously, and the tissue vaporizes. If the focal point of the beam is moved over the tissue, an incision results of a depth that depends on the intensity of the beam and on the speed of the movement. The incision is relatively blood- 